
\title{
25 Research Soure \\ Label-Free LC-MS/MS Proteomics Analyses Reveal Proteomic Changes In Oxidative Stress And The SOD Antioxidant Strategy In TM Cells.
}

\section{Qian Li}

The Second Affiliated Hospital of Anhui Medical University

\section{Liyu Zhang}

The Second Affiliated Hospital of Anhui Medical University

Yuxin Xu ( $\sim$ xuyuxin1168@sina.com)

The Second Affiliated Hospital of Anhui Medical University https://orcid.org/0000-0001-7138-4265

\section{Research Article}

Keywords: Trabecular meshwork cells, Oxidative stress, Label-free, Proteomic analysis, SOD.

Posted Date: December 17th, 2021

DOI: https://doi.org/10.21203/rs.3.rs-1055533/v1

License: (c) (i) This work is licensed under a Creative Commons Attribution 4.0 International License.

Read Full License 


\section{Abstract}

Background: Treatment for glaucoma has traditionally been limited to reducing intraocular pressure (IOP). Inhibiting oxidative stress in the trabecular meshwork (TM) is regarded as a new treatment for glaucoma; however, the effects do not meet expectations. Exploring the mechanism by which oxidative stress and antioxidant stress occur in TM cells will offer clues to aid the development of new treatments.

Methods and results: In our study, we cultured TM cells and used $\mathrm{H}_{2} \mathrm{O}_{2}$ and SOD to induce and inhibit oxidative stress, respectively. Label-free LC-MS/MS quantitative proteomic analysis was conducted to analyze the differentially expressed proteins and relevant signaling pathways. A total of 24 upregulated proteins and 18 downregulated proteins were identified under oxidative stress. PTGS2, TGF $\beta 22$ and ICAM1 were the key proteins. The PTGS2/NF-kb pathway, TGF- $\beta$ /Smad signaling pathway and AGE-RAGE signaling pathway in diabetic complications may be the major signaling pathways under conditions of ROS-induced damage in TM cells. Seventy-eight proteins were upregulated and 73 proteins were downregulated under antioxidant stress in TM cells. The key proteins included collagen family proteins, which were upregulated, and ICAM-1, which was downregulated. The ECM-receptor interaction pathway was the most important pathway under antioxidant stress.

Conclusions: Key proteins and signaling pathways play important roles in the mechanisms of oxidative stress and antioxidant strategies in TM cells. ICAM-1 knockdown can suppress the apoptosis of TM cells induced by $\mathrm{H}_{2} \mathrm{O}_{2}$, which may reveal new therapeutic targets and biomarkers for glaucoma.

\section{Introduction}

Glaucoma is a multi-factorial optic neuropathy characterized by irreversible vision loss and corresponding atrophy of the optic nerve. It has been estimated to affect $3.5 \%$ of individuals over 40 years old and projected to affect a total of 112 million people by $2040^{[1}$. Although the pathogenesis of glaucoma is not fully understood, the trabecular meshwork (TM) is regarded as the key factor for the worsening of glaucoma. The TM can reduce aqueous humor outflow, leading to the high-tension glaucoma cascade ${ }^{[2}$. Moreover, it has been hypothesized that glaucomatous TM cells (TMCs) may have altered gene and protein expression, generating molecular signals and contributing to retinal ganglion cell (RGC) death in both normal-tension and high-tension glaucoma ${ }^{33}$. Therefore, understanding the mechanisms of pathological changes in the TM is critical for the prevention and treatment of glaucoma.

Oxidative stress (OS) plays an important role in the pathological mechanism of glaucoma ${ }^{[4}$, as the associated increases in reactive oxygen species (ROS) can damage the cornea, iris, and TM. Thus, focusing solely on reducing intraocular pressure (IOP) for glaucoma therapy, especially for some patients who do not respond to this type of treatment, is insufficient ${ }^{[5}$. Notably, reducing $O S$ is emerging as a therapeutic strategy for glaucoma ${ }^{[6}$. Some papers have reported the use of antioxidant drug treatment or OS inhibition for the treatment of glaucoma; however, the effects have not met the expectations ${ }^{[7,8}$ because the underlying molecular mechanisms are far from elucidated. Izzotti et al ${ }^{[9}$ reported that the TM 
is the most sensitive tissue in the eye. When OS occurs, TM mitochondrial dysfunction, inflammatory cytokine release, and impairment of extracellular matrix (ECM) components may occur ${ }^{[4,10}$. Therefore, exploration of how ROS damage the TM and exactly how OS can be suppressed is crucial for further investigating treatments for glaucoma.

Proteins are major agents in the execution of various physiological and pathological processes. When OS occurs, some specific proteins produced by TMCs may participate in the pathological pathway, causing the glaucoma cascade. Therefore, detecting and quantifying the proteins that are changed in TMCs under OS will offer evidence to elucidate the pathogenesis. Thus far, label-free methods have been widely used in clinical research, biomarker discovery, and personalized medicine ${ }^{[11}$. Compared with other proteomics technologies, these methods are more convenient, versatile, and flexible ${ }^{[12}$. In this study, we cultured human TMCs (HTMCs) and used $\mathrm{H}_{2} \mathrm{O}_{2}$ to induce OS. We also added superoxide dismutase (SOD), the first-line antioxidant, to inhibit OS in HTMCs. Furthermore, we used label-free techniques to detect the differentially expressed proteins (DEPS) in different groups. Through bioinformatics analysis, we sought to identify the key proteins and their related signaling pathways in order to explore the mechanisms in TMCs under OS or antioxidant stress.

\section{Materials And Methods}

\subsection{Cell culture and identification}

Primary explant-derived HTMCs (iCell Bioscience Inc, China) were grown in DMEM/F12 with 20\% serum and kept at $37^{\circ} \mathrm{C}$ in a $5 \% \mathrm{CO}_{2}$ environment. Only cells in the $3^{\text {rd }}$ to $5^{\text {th }}$ passage were used. To identify TMCs, collagen type IV, laminin and fibronectin antigens were detected by immunocytochemistry.

\subsection{Sample collection and protein preparation}

TMCs were pretreated with $\operatorname{SOD}(0,1,5$, or $10 \mathrm{U} / \mathrm{mL})$ for 30 min and then exposed for 24 hours to a range of $\mathrm{H}_{2} \mathrm{O}_{2}$ concentrations $(0,50,100,150$, and $200 \mu \mathrm{M})$. The concentrations of $\mathrm{H}_{2} \mathrm{O}_{2}$ and SOD in the group with the most obvious protective effect of SOD were screened through Cell Counting Kit-8 (CCK-8) assay. The cells were divided into three groups: the control group (without any interference, group $\mathrm{C}$ ), the $\mathrm{H}_{2} \mathrm{O}_{2}$-treated group (with exposure to $\mathrm{H}_{2} \mathrm{O}_{2}$ only, group $\mathrm{H}$ ), and the SOD pretreatment group (with exposure to SOD before $\mathrm{H}_{2} \mathrm{O}_{2}$ addition, group $\mathrm{S}$ ). The cells were collected and stored at $-80^{\circ} \mathrm{C}$ after homogenization and centrifugation. Then, a mammalian tissue total protein extraction kit (AP0601-50) was used to extract proteins from the different groups. The protein concentrations were estimated using a Bradford assay kit. Next, $20 \mu \mathrm{g}$ of protein from each sample was mixed with $5 \times$ loading buffer at a ratio of $5: 1$ $(\mathrm{v} / \mathrm{v})$. The supernatant was collected after $5 \mathrm{~min}$ of boiling in a water bath and $10 \mathrm{~min}$ of centrifugation at $14,000 \times \mathrm{g}$. The proteins were separated on a 10\% SDS-PAGE gel (constant current of $14 \mathrm{~mA}, 90 \mathrm{~min}$ ). Coomassie Blue R-250 staining was used to visualize the protein bands. After quantification, $60 \mu \mathrm{g}$ of protein from each sample was mixed with DTT $(5 \mu \mathrm{l}$ of $1 \mathrm{M})$ and incubated at $37^{\circ} \mathrm{C}$ for 1 hour. Then, iodoacetamide (IAA, $20 \mu \mathrm{l}$ of $1 \mathrm{M}$ ) was added, and the samples were incubated for 1 hour in the dark. All 
solutions were then transferred to a centrifuge tube, and the supernatant was discarded after centrifugation at $14000 \times \mathrm{g}$ for $20 \mathrm{~min}$ at $4^{\circ} \mathrm{C}$. The filter was centrifuged after the addition of $100 \mu \mathrm{l} \mathrm{UA}$ buffer (8 M urea, $100 \mu \mathrm{M}$ Tris- $\mathrm{HCl}, \mathrm{pH} 8.0$ ); this step was repeated twice. After that, we added $100 \mu \mathrm{l}$ of $\mathrm{NH} 4 \mathrm{HCO} 3(50 \mathrm{mM})$ to the proteins, mixed the samples and centrifuged them under the same conditions. The supernatant was removed as before; this step was repeated three times. Finally, we replaced the collection tube with a new collection tube, added trypsin at a ratio of 50:1 (protein:trypsin) to the digested proteins and incubated the samples at $37^{\circ} \mathrm{C}$ for 16 hours. After digestion, the peptides were vacuum dried, dissolved in $0.1 \%$ trifluoroacetic acid (TFA), desalted on C18 cartridges (Empore SPE Cartridges C18, standard density), dried by vacuum centrifugation and reconstituted in formic acid (FA).

\subsection{LC-MS/MS analysis}

Each sample was separated by HPLC. After loading onto a C18 column, the samples were separated by a linear gradient formed from buffer $A\left(0.1 \% \mathrm{FA} / \mathrm{H}_{2} \mathrm{O}\right)$ and buffer $\mathrm{B}(0.1 \% \mathrm{FA} /$ acetonitrile $(\mathrm{ACN}))$ at a flow rate of $600 \mathrm{nl} / \mathrm{min}$. The chromatographic separation gradient is shown in Table 1. After separation, each sample was analyzed by mass spectrometry (MS) with an Orbitrap Fusion Lumos Mass Spectrometer (Thermo Scientific). The MS scan mass window was set at 300-1400 m/z with the 20 most intense signals per cycle fragmented. The automatic gain control (AGC) target was set to 500000 , and the maximum injection time was set to $50 \mathrm{~ms}$. Each sample was analyzed in triplicate.

\subsection{Data analysis and bioinformatics analysis}

MaxQuant (1.6.17) was used to search the reviewed FASTA database in UniProt with Homo sapiens as the organism. The protein expression data are presented in a heatmap. The DEPs between groups were defined as significantly upregulated or downregulated on the basis of a fold change $(F C) \geq 1.5$ and $p<0.05$ (upregulated) or a FC $\leq 0.667$ and $p<0.05$ (downregulated) (experimental group/control group). We used Metascape, a web-based resource (http://metascape.org), to conduct Gene Ontology (GO) analysis and used the Kyoto Encyclopedia of Genes and Genomes (KEGG) Orthology-Based Annotation System (KOBAS) online analysis tool (http://kobas. cbi.pku.edu.cn/) to perform KEGG pathway analyses. Database enrichment analysis was performed using the UniProtKB database (Release 2016 10). GO enrichment included three ontologies (biological process (BP), molecular function (MF), and cellular component (CC)). In addition, we performed protein-protein interaction (PPI) analysis using STRING software (http://string-db.org/) and then imported the results into Cytoscape software (http://www.cytoscape.org/, version 3.8.2) to further analyze functional PPI networks. EVenn (http://www.ehbio.com/test/venn/\#/) was used to create Venn diagrams.

\subsection{Cell transfection with siRNAs}

SiRNA against intracellular adhesion molecule-1 (ICAM-1) (silCAM-1) was provided by GenePharma (Shanghai, China) and transfected into TMCs using Lipofectamine 2000 reagent after induction with $\mathrm{H} 2 \mathrm{O} 2$ for 24 hours. The sense strand of silCAM-1 used for gene knockdown was as follows:

5'- GCCAACCAAUGUGCU AUUCAAdTdT-3'. The scrambled siRNA sequence 
was UUCUCCGAACGUGUCACGUdTdT. Before transfection, the TMCs were cultured in 6-well plates with complete medium for 24 hours. silCAM-1 was transfected with Lipofectamine 2000 (Thermo) in serumfree DMEM for 6 hours, and then the mixture was replaced with complete medium. Western blotting was used to verify protein knockdown 24 hours post transfection.

\subsection{Western blotting}

TMCs were lysed with lysis buffer $(20 \mathrm{mmol} / \mathrm{L}$ HEPES, $150 \mathrm{mmol} / \mathrm{L} \mathrm{NaCl}, 1 \mathrm{mmol} / \mathrm{L} \mathrm{EGTA}, 1 \mathrm{mmol} / \mathrm{L}$ EDTA, $10 \%$ glycerol, $1 \mathrm{mmol} / \mathrm{L} \mathrm{MgCl}_{2}, 1 \%$ Triton X-100). The extracts were centrifuged at $12,000 \mathrm{rpm}$ for $20 \mathrm{~min}$ at $4^{\circ} \mathrm{C}$, and the supernatants were collected. The protein extracts were separated on $10 \%$ polyacrylamide-SDS gels, transferred to PVDF membranes and then blocked with $5 \%$ skimmed milk powder for 1 hour. After incubation with primary antibodies (anti-ICAM, Abcam) overnight, the membranes were washed three times and incubated with fluorescent secondary antibodies at room temperature for 2 hours. The fluorescent signals were captured using an infrared imager (Millipore, USA).

\subsection{Cell viability assay}

TMC viability was examined using a CCK-8 (Dojindo Molecular Technologies, Gaithersburg, MD, USA). TMCs were seeded in 96 -well plates and incubated at $37^{\circ} \mathrm{C}$ for 24 hours. After cell transfection and/or stimulation with $\mathrm{H} 2 \mathrm{O} 2$, the culture medium was replaced with TMC medium (TMCM) containing $10 \%$ CCK-8 solution, and the cells were incubated at $37^{\circ} \mathrm{C}$ for an additional 2 hours. Finally, the absorbance at $450 \mathrm{~nm}$ was detected by using a microplate reader (Bio-Rad, Hercules, CA, USA).

\subsection{Statistical analysis}

Two-tailed Student's t-test was used for statistical analysis. All data are expressed as the mean \pm $\mathrm{SE}$, and a $p$ value $<0.05$ was considered to indicate statistical significance.

\section{Results}

\subsection{Cell identification and model establishment}

The results of immunocytochemistry for cells with collagen type IV, laminin and fibronectin antigens are shown in Figure 1. We observed that these antigens were all expressed in the cells, which confirmed that the cells we cultured were TMCs. The CCK-8 results are also shown in Figure 2. When the concentration of $\mathrm{H}_{2} \mathrm{O}_{2}$ was $150 \mu \mathrm{M}$ and the concentration of SOD was increased to $5 \mathrm{U} / \mathrm{mL}$, the numbers of cells significantly differed between the $\mathrm{H}_{2} \mathrm{O}_{2}$-treated group and the SOD-pretreated group. Hence, we determined that the $\mathrm{H}_{2} \mathrm{O}_{2}$-treated group (group $\mathrm{H}$ ) represented TMCs cultured with $150 \mu \mathrm{M} \mathrm{H}_{2} \mathrm{O}_{2}$ for 24 hours, and the SOD-pretreated group (group S) represented TMCs pretreated with $5 \mathrm{U} / \mathrm{mL}$ SOD for $30 \mathrm{~min}$ and then subjected to the same conditions as the $\mathrm{H}_{2} \mathrm{O}_{2}$-treated group. 


\subsection{LC-MS/MS analysis and identification of DEPs}

Each groups included 3 samples. After LC-MS/MS analysis, we identified 24 upregulated proteins and 18 downregulated proteins between group $\mathrm{H}$ and

group C, (Figure $3 \mathrm{~A}, \mathrm{~B}$ ) and we identified 78 upregulated proteins and 73 downregulated proteins between group $S$ and group $\mathrm{H}$ (Figure $3 \mathrm{C}, \mathrm{D}$ ).

\subsection{GO functional annotation and enrichment analysis}

GO functional annotation was performed based on three categories; BP, MF and CC (Figure 4).We found that between group $\mathrm{H}$ and group $\mathrm{C}$ (Figure $4 \mathrm{~A}$ and $\mathrm{B}$ ), the major $\mathrm{GO}$ terms were related to "cellular process" (up: $n=23$, down: $n=18$ ) and "biological regulation" (up: $n=17$, down: $n=11$ ) in the BP category, "cellular anatomical entity" (up: $n=23$, down: $n=17$ ) in the CC category and "binding" (up:

$n=22$, down: $n=16$ ) in the MF category. Furthermore, between group $S$ and group $H$ (Figure $4 C$ and $D$ ), the major GO terms were the same as those between group H and group C: "cellular process" (up: $n=68$, down: $n=70$ ) and "biological regulation" (up: $n=50$, down: $n=50$ ) for the BP category, "cellular anatomical entity" (up: $n=75$, down: $n=71$ ) for the CC category and "binding" (up: $n=64$, down: $n=68$ ) for the MF category. In addition, the results of GO functional enrichment analysis are shown in Figure 5 . The enriched DEPs between group $\mathrm{H}$ and group $\mathrm{C}$ (Figure $5 \mathrm{~A}$ ) were related mainly to the functional terms "regulation of plasma membrane-bounded cell projection assembly", "negative regulation of blood coagulation" and "regulation of cell projection assembly", whereas the enriched DEPs between group $S$ and group H (Figure 5 B) were related primarily to the functional terms "collagen trimer", "extracellular matrix organization" and "extracellular structure organization".

\subsection{PPI analysis and Venn analysis}

To determine the molecular mechanisms by which OS and antioxidant stress occur in TMCs, we used STRING software combined with Cytoscape software to analyze the DEPs in the different groups. Figure 6 A shows the key PPI network for the upregulated DEPs between group $\mathrm{H}$ and group C. Figure $6 \mathrm{~B}$ shows the key PPI network for the downregulated DEPs between group $\mathrm{S}$ and group $\mathrm{H}$. Furthermore, to determine the mechanism by which SOD protects TMCs, we conducted Venn analyses to identify the key proteins (Figure 7).

\subsection{KEGG pathway analysis for DEPs}

We conducted KEGG signaling pathway analysis for DEPs. The results of KEGG pathway enrichment between group $\mathrm{H}$ and group $\mathrm{C}$ are shown in Figure $8 \mathrm{~A}$. The top four pathways in KEGG enrichment were the TNF signaling pathway (major proteins: ICAM-1 and prostaglandin-endoperoxide synthase 2 (PTGS2)), the NF-kB signaling pathway (major proteins: ICAM-1 and PTGS2), adherens junctions (major proteins: BAIAP2 and TGF $\beta r 2$ ) and the AGE-RAGE signaling pathway in diabetic complications (major proteins: ICAM-1 and TGF $\beta$ r2). We deemed the AGE-RAGE signaling pathway in diabetic complications noteworthy after our KEGG pathway annotation and enrichment analyses (Figure 9). The results of KEGG 
pathway analysis for the downregulated DEPs between group $\mathrm{S}$ and group $\mathrm{H}$ are shown in Figure $8 \mathrm{~B}$. The top three pathways were mineral absorption (major proteins: ATOX1 and CYBRD1), African trypanosomiasis (major proteins: ICAM1 and FAS), shown in Figure 10 and central carbon metabolism in cancer (major proteins: GLS and G6PD)

\subsection{ICAM-1 knockdown rescues the viability of TMCs treated with $\mathrm{H}_{2} \mathrm{O}_{2}$}

To further study the regulation of ICAM-1 in H2O2-treated TMCs, we transfected siRNA into TMCs and used a CCK-8 assay to test TMC viability. The expression of ICAM-1 in the H2O2-treated group was higher than that in blank group, and the expression in the group transfected with silCAM-1 was lower than that in the H2O2-treated group (Figure $11 \mathrm{~A}$ ). Moreover, the viability of TMCs in the different groups was obviously different over time. After treatment with $\mathrm{H} 2 \mathrm{O} 2$, viability was reduced, while viability recovered after transfection with silCAM-1 compared to the H2O2-treated group (Figure 11 B).

\section{Discussion}

Glaucoma is a chronic progressive optic neuropathy characterized by irreversible damage to the retinal nerve fiber layer (RNFL) as well as peripheral, and occasional central, vision loss. Elevated IOP is the major risk factor for glaucoma; hence, lowering IOP is regarded as the first-line treatment. However, the efficacy of IOP reduction is undoubtedly insufficient; furthermore, normal-tension glaucoma exists. Sacca et al ${ }^{[3}$ reported that OS in TMCs affects gene and protein expression, contributing to the development of glaucoma. In our study, we cultured TMCs and compared the differentially expressed proteins through label-free LC-MS/MS proteomics analyses to identify the key proteins and pathways in cells under OS and antioxidant stress. Finally, a list of key proteins was identified, and the major pathways were explored.

Figure 1 shows the results of cell identification. The expression of collagen type IV, laminin and fibronectin demonstrated that the cells we cultured were TMCs. As shown in Figure 2, we found that apoptosis increased as the $\mathrm{H}_{2} \mathrm{O}_{2}$ concentration increased. SOD had a protective effect on TMCs; however, when the concentration of $\mathrm{H}_{2} \mathrm{O}_{2}$ reached $200 \mu \mathrm{M}$, the apoptosis of TMCs increased. Moreover, when the concentration of SOD reached $10 \mathrm{U} / \mathrm{mL}$, SOD had a harmful effect on TMCs. We determined that the difference was most significant when the concentration of $\mathrm{H}_{2} \mathrm{O}_{2}$ was $150 \mu \mathrm{M}$ and the concentration of SOD was $5 \mathrm{U} / \mathrm{mL}$. Hence, we selected these concentrations for further experiments.

Our study included three groups: group $\mathrm{C}$, group $\mathrm{H}$ and group $\mathrm{S}$. We used label-free LC-MS/MS proteomics analyses to analyze the DEPs. After comparing group $\mathrm{H}$ and group $\mathrm{C}$, we obtained 24 upregulated proteins and 14 downregulated proteins, as shown in Figure $3 \mathrm{~A}$ and $\mathrm{B}$. We obtained 8 upregulated proteins and 73 downregulated proteins between group $S$ and group $\mathrm{H}$. After GO, KEGG and PPI analyses, we ultimately obtained a list of key proteins and pathways that play important roles in the molecular mechanisms in TMCs under OS and antioxidant stress. 
$\mathrm{H}_{2} \mathrm{O}_{2}$ is often used to induce OS. In our study, we cultured TMCs with $150 \mu \mathrm{M} \mathrm{H}_{2} \mathrm{O}_{2}$ for 24 hours. Among the upregulated proteins, PPI analyses revealed PTGS2, TGF $\beta$ r2 and ICAM-1 as important DEPs between group $\mathrm{H}$ and group $\mathrm{C}$. Identification of these proteins suggests the mechanism by which OS damages TMCs.

The levels of PTGS2 (also known as COX2), a rate-limiting enzyme in the conversion of arachidonic acid into prostaglandins, often increase during inflammation. In a study by Li et al ${ }^{[13}$, melanocytes were treated with $\mathrm{H}_{2} \mathrm{O}_{2}$, RNA was extracted, and the differential expression profiles of RNAs were detected and analyzed through GO and KEGG analyses. Li et al also concluded that PTGS2 might play central regulatory roles in the OS response. OS often damages cells by increasing apoptosis and inflammation. PTGS2 is a key enzyme involved in inflammation that can activate the PI3-K/AKT and PKA/CREB pathways, which facilitate apoptosis and inflammation ${ }^{[14}$. Furthermore, PTGS2 is an NF-kB target gene whose promoter has been shown to contain several binding sequences for the transcription factor NF$\mathrm{kB}^{[15}$. The NF-KB pathway can activate the expression of PTGS2, and a growing body of evidence suggests that the NF-KB pathway participates in the inflammatory and immune responses, activating the expression of adhesion molecules, cytokine receptors, and numerous cytokines, which effectively affects the process of apoptosis ${ }^{[16}$. In our study, the NF-KB pathway was identified as the major KEGG pathway in the upregulated DEPs between group $\mathrm{H}$ and group $\mathrm{C}$. It can be concluded that NF-KB stimulates PTGS2 expression and may be largely responsible for the damage to TMCs after treatment with $\mathrm{H}_{2} \mathrm{O}_{2}$.

Transforming growth factor $\beta$ (TGF- $\beta$ ) exists in three isoforms, TGF $\beta 1$, TGF $\beta 2$, and TGF $\beta 3$. Numerous studies have demonstrated that TGF- $\beta$ increases ROS production and suppresses the antioxidant system, thereby inducing OS. It can activate cells to produce ROS via NADPH oxidase ${ }^{[17}$ leading to apoptosis. Active TGF- $\beta$ binding to TGF $\beta 2$ on the cell membrane activates TGF $\beta R 1$, initiating the TGF $\beta$ signaling pathway and regulating cell hypertension, proliferation, apoptosis, differentiation, and morphogenesis. The TGF- $\beta$ /Smad signaling pathway is an important downstream signal transduction pathway of TGF- $\beta$ mediated apoptosis. There are eight Smad proteins, and Smad2 and Smad3 belong to the receptorregulated Smad (R-Smad) family. When TGF $\beta 1$ is activated, heterogeneous complexes are formed; Smad2 and Smad3 are then phosphorylated and can bind with Smad4 (the comediator Smad, Co-Smad) [18. The Smad complexes are translocated into the nucleus, where they regulate the transcription of target genes and the production of ECM proteins ${ }^{[18}$, which has a negative effect on cell growth. Furthermore, TGF- $\beta$ plays a pivotal role in the development of fibrosis ${ }^{[19}$. Zhang et al ${ }^{[20}$ used DZ2002 to suppress the TGF- $\beta /$ Smad signaling pathway in order to decrease fibrosis in human dermal fibroblasts under conditions of systemic sclerosis. In our study, TGF $\beta$ r2 was increasingly expressed in TMCs, and the TGF$\beta /$ Smad signaling pathway was involved in the AGE-RAGE signaling pathway in diabetic complications (Figure 8), leading to thrombogenesis, inflammation and atherosclerosis. We can conclude that the TGF$\beta /$ Smad signaling pathway may enhance apoptosis of TMCs or induce fibrosis in TMCs to affect TMC function. One of the most important proteins involved in the TGF- $\beta /$ Smad signaling pathway is ICAM- 1 . ICAM-1, a member of the immunoglobulin superfamily, is often expressed at low levels by endothelial cells and is highly expressed and induced by a variety of inflammatory cytokines when OS occurs. In 
endothelial cells, ICAM-1 often plays a key role in mediating firm adhesion of leukocytes and participates in many physiological processes ${ }^{[21}$. It has been reported that ICAM-1 can regulate endothelial cell permeability in healthy and inflamed tissue ${ }^{[22,23}$. When ICAM-1 levels increase, JNK is activated, leading to internalization of VE-cadherin, disruption of cell junctions and impairment of cell barrier function ${ }^{[21}$. In addition, ICAM- 1 can activate monocytes to enhance the stimulation and transmigration of inflammatory cells into the ECM) ${ }^{[24,25}$, substantially increasing apoptosis. In our study, ICAM-1 levels were significantly increased, and ICAM-1 was the major protein in the TNF signaling pathway, NF-kB signaling pathway and AGE-RAGE signaling pathway in diabetic complications. These pathways were all major KEGG pathways in TMCs under OS that were demonstrated to be obviously related to apoptosis. In summary, ICAM-1 levels increased when OS occurred in TMCs in our study, and ICAM-1 participated in the PTGS2/NF-kb pathway, TGF- $\beta$ /Smad signaling pathway and AGE-RAGE signaling pathway in diabetic complications to reduce the viability of TMCs.

SOD is the first-line antioxidant and can effectively eliminate ROS. Jiang et al ${ }^{[26}$ demonstrated that adeno-associated virus (AAV)-mediated pretreatment with SOD2 is able to attenuate OS. In our study, we pretreated TMCs with SOD for $30 \mathrm{~min}$ and then exposed the TMCs to $150 \mu \mathrm{M} \mathrm{H}_{2} \mathrm{O}_{2}$. After conducting Venn analyses, we found that ICAM- 1 was also the key DEP whose expression was downregulated after SOD was added to TMCs. As discussed previously, increased ICAM- 1 is one of the most important factors that damage TMCs under OS; What's more,ICAM-1 is also the key protein involved in African trypanosomiasis, one of the major KEGG pathway for downregulated DEPs between group $S$ and group $\mathrm{H}$. hence, we can conclude that SOD may suppress the OS induced by $\mathrm{H}_{2} \mathrm{O}_{2}$ by decreasing ICAM- 1 levels to reduce the apoptosis of TMCs.

To further study the function of ICAM-1, we reduced the expression of ICAM- 1 via transfection of silCAM1. Western blotting was used to test the expression of ICAM-1, and a CCK-8 assay was used to measure the viability of TMCs. We found that after treatment with $\mathrm{H} 2 \mathrm{O} 2$, the expression of ICAM- 1 in TMCs was elevated, while transfection with silCAM-1 inhibited ICAM-1 expression and increased viability over time. All the results illustrate that oxidative injury of human TMCs induced by $\mathrm{H} 2 \mathrm{O} 2$ is related to ICAM-1 expression and that knockdown of ICAM-1 attenuates the injury induced by $\mathrm{H} 2 \mathrm{O} 2$.

\section{Conclusion}

In this study, we used label-free LC-MS/MS proteomics analyses to analyze the proteomic changes that occurred when TMCs were subjected to $\mathrm{OS}$ induced by $\mathrm{H}_{2} \mathrm{O}_{2}$ and antioxidant stress induced by SOD. The results of GO analysis, KEGG analysis and PPI network analysis indicated that the upregulated proteins PTGS2, TGFBr2 and ICAM-1 were the key proteins that participate in the PTGs2/NF-kb pathway, TGF$\beta /$ Smad signaling pathway and AGE-RAGE signaling pathway in diabetic complications, leading to the apoptosis and fibrosis of TMCs. SOD may protect TMCs majorly by decreasing the levels of ICAM-1, which participate in African trypanosomiasis inhibiting apoptosis. Notably, higher expression of ICAM-1 
was associated with lower viability of TMCs. These data provide valuable insights into the roles of these key proteins and pathways, which may be regarded as new therapeutic targets for glaucoma.

\section{Abbreviations}

TM, trabecular meshwork; HTMC, human trabecular meshwork cell; IOP, intraocular pressure, LC-MS/MS, liquid chromatography-tandem mass spectrometry; SOD, superoxide dismutase; PTGS2, prostaglandinendoperoxide synthase; TGF $\beta$ r2, transforming growth factor $\beta$ receptor 2; ICAM-

1, intercellular adhesion molecule 1; OS, oxidative stress; ROS, reactive oxygen species; ECM, extracellular matrix; DEP, differentially expressed protein.

\section{Declarations}

Ethics approval and consent to participate

Not applicable.

Consent for publication

Yes.

Availability of data and materials

Not applicable.

Competing interests

The authors declare that they have no competing interests.

\section{Funding}

Anhui Provincial key research and technology development program (9101120202).

\section{Authors' contributions}

Qian Li conducted experiments, analysed data and drafted the manuscript. Liyu Zhang participated in the design and coordination, Yuxin Xu participate in the manuscript review, All authors read and approved the final manuscript.

\section{Acknowledgements}

The authors thank anyone offer helpful suggestions during the experiment.

\section{References}


1. Yap TE, Davis BM, Guo L et al. Annexins in Glaucoma. Int J Mol Sci. 2018; 19(4).

2. Sacca SC, Gandolfi S, Bagnis A et al. The Outflow Pathway: A Tissue With Morphological and Functional Unity. J Cell Physiol. 2016; 231(9):1876-1893.

3. Sacca SC, Gandolfi S, Bagnis A et al. From DNA damage to functional changes of the trabecular meshwork in aging and glaucoma. Ageing Res Rev. 2016; 29:26-41.

4. Kim SH, Kim H. Inhibitory Effect of Astaxanthin on Oxidative Stress-Induced Mitochondrial Dysfunction-A Mini-Review. Nutrients 2018; (10):1137.

5. Osborne NN, del Olmo-Aguado S. Maintenance of retinal ganglion cell mitochondrial functions as a neuroprotective strategy in glaucoma. Curr Opin Pharmacol. 2013; 13(1):16-22.

6. Kimura A, Namekata K, Guo X et al. Targeting Oxidative Stress for Treatment of Glaucoma and Optic Neuritis. Oxid Med Cell Longev. 2017; 2017:2817252.

7. Inman DM, Lambert WS, Calkins DJ et al. alpha-Lipoic acid antioxidant treatment limits glaucomarelated retinal ganglion cell death and dysfunction. PLoS One. 2013; 8(6):e65389.

8. Iraha S, Hirami Y, Ota S et al. Efficacy of valproic acid for retinitis pigmentosa patients: a pilot study. Clin Ophthalmol. 2016; 10:1375-1384.

9. Izzotti A, Sacca SC, Longobardi M et al. Sensitivity of ocular anterior chamber tissues to oxidative damage and its relevance to the pathogenesis of glaucoma. Invest Ophthalmol Vis Sci. 2009; 50(11):5251-5258.

10. Wang $M$, Zheng Y. Oxidative stress and antioxidants in the trabecular meshwork. PeerJ. 2019; 7:e8121.

11. Jiang Y, Sun A, Zhao Y et al. Proteomics identifies new therapeutic targets of early-stage hepatocellular carcinoma. Nature. 2019; 567(7747):257-261.

12. Zhao $L$, Cong $X$, Zhai $L$ et al. Comparative evaluation of label-free quantification strategies. $J$ Proteomics. 2020; 215:103669.

13. Li S, Zeng $\mathrm{H}$, Huang $\mathrm{J}$ et al. Identification of the Competing Endogenous RNA Networks in Oxidative Stress Injury of Melanocytes. DNA Cell Biol. 2021; 40(2):192-208.

14. Yang H, Wang H, Shu Y et al. miR-103 Promotes Neurite Outgrowth and Suppresses Cells Apoptosis by Targeting Prostaglandin-Endoperoxide Synthase 2 in Cellular Models of Alzheimer's Disease. Front Cell Neurosci. 2018; 12:91.

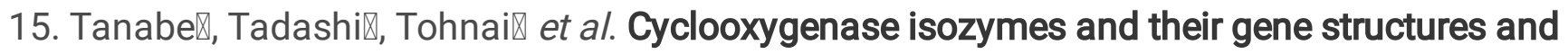
expression. Elsevier Science Inc. 2002; 68-69:95-114. 
16. Zhou Z, Lu C, Meng S et al. Silencing of PTGS2 exerts promoting effects on angiogenesis endothelial progenitor cells in mice with ischemic stroke via repression of the NF-kappaB signaling pathway. $J$ Cell Physiol. 2019; 234(12):23448-23460.

17. Cai H, Su S, Li Y et al. Protective effects of Salvia miltiorrhiza on adenine-induced chronic renal failure by regulating the metabolic profiling and modulating the NADPH oxidase/ROS/ERK and TGF-beta/Smad signaling pathways. J Ethnopharmacol. 2018; 212:153-165.

18. Shi Y, Massagué J. Mechanisms of TGF- $\beta$ Signaling from Cell Membrane to the Nucleus. Cell. 2003; 113(6):685-700.

19. Liu RM, Desai LP. Reciprocal regulation of TGF-beta and reactive oxygen species: A perverse cycle for fibrosis. Redox Biol. 2015; 6:565-577.

20. Zhang Z, Wu Y, Wu B et al. DZ2002 ameliorates fibrosis, inflammation, and vasculopathy in experimental systemic sclerosis models. Arthritis Res Ther. 2019; 21(1):290.

21. Bui TM, Wiesolek HL, Sumagin R. ICAM-1: A master regulator of cellular responses in inflammation, injury resolution, and tumorigenesis. J Leukoc Biol. 2020; 108(3):787-799.

22. Sumagin R, Lomakina E, Sarelius IH. Leukocyteendothelial cell interactions are linked to vascular permeability via ICAM-1-mediated signaling. Am J Physiol Heart Circ Physiol. 2008; 295(3):H969-H977.

23. Pfeiffer F, Kumar V, Butz $S$ et al. Distinct molecular composition of blood and lymphatic vascular endothelial cell junctions establishes specific functional barriers within the peripheral lymph node. Eur $J$ Immunol. 2008; 38(8):2142-2155.

24. Yeh MC, Wu BJ, Li Y et al. BT2 Suppresses Human Monocytic-Endothelial Cell Adhesion, Bone Erosion and Inflammation. $J$ Inflamm Res. 2021; 14:1019-1028.

25. Avola R, Granata G, Geraci C et al. Oregano (Origanum vulgare L.) essential oil provides antiinflammatory activity and facilitates wound healing in a human keratinocytes cell model. Food Chem Toxicol. 2020; 144:111586.

26. Jiang. W, Tang. L, Jun Zeng BC. Adeno-associated virus mediated SOD

gene therapy protects the retinal ganglion cells from chronic intraocular pressure elevation induced injury via attenuating oxidative stress and improving mitochondrial dysfunction in a rat model. Am J Trans/ Res. 2016; 8 (2):799-810

\section{Tables}

Table 1 Chromatographic separation gradient for LC-MS/MS Analysis 


\begin{tabular}{|lllllll|}
\hline Time & 0 & 11 & 48 & 68 & 69 & 75 \\
\hline A\% & $93 \%$ & $85 \%$ & $75 \%$ & $60 \%$ & $0 \%$ & $0 \%$ \\
\hline B\% & $7 \%$ & $15 \%$ & $25 \%$ & $70 \%$ & $100 \%$ & $100 \%$ \\
\hline
\end{tabular}

Table2.top 5 decreased DEPs between group $\mathrm{S}$ and group $\mathrm{H}$

\begin{tabular}{lllll}
\hline Accession & Description & Gene names & Groups/H. fold change & $P$ Value \\
\hline P05362 & $\begin{array}{l}\text { Intercellular adhesion } \\
\text { molecule 1 }\end{array}$ & ICAM1 & 11.14303431 & 0.029869 \\
\hline P62879 & $\begin{array}{l}\text { Guanine nucleotide-binding } \\
\text { protein }\end{array}$ & GNB2 & 10.20115299 & 0.002779 \\
\hline P50416 & $\begin{array}{l}\text { Carnitine 0- } \\
\text { palmitoyltransferase 1 }\end{array}$ & CPT1A & 8.228885209 & 0.025462 \\
\hline Q5EBL4 & RILP-like protein 1 & RILPL1 & 7.213202152 & 0.005149 \\
Q9NRX5 & Serine incorporator 1 & SERINC1 & 7.164497945 & 0.015199 \\
\hline
\end{tabular}

\section{Figures}

\section{Image not available with this version}

Figure 1

This image is not available with this version.

\section{Image not available with this version}

\section{Figure 2}

This image is not available with this version. 


\section{Image not available with this version}

Figure 3

This image is not available with this version.

Figure 4

This image is not available with this version.

\section{Image not available with this version}

Figure 5

This image is not available with this version.

\section{Image not available with this version}

Figure 6

This image is not available with this version. 


\section{Figure 7}

This image is not available with this version.

\section{Figure 8}

This image is not available with this version.

\section{Figure 9}

This image is not available with this version.

Figure 10

This image is not available with this version.

\section{Image not available with this version}

\section{Figure 11}

This image is not available with this version. 